\title{
The Numerical Computation of the Critical Boundary Displacement for Radial Cavitation
}

\author{
Pablo V. Negrón-Marrero* \\ Department of Mathematics \\ University of Puerto Rico \\ Humacao, PR 00791-4300
}

\author{
Jeyabal Sivaloganathan ${ }^{\dagger}$ \\ Department of Mathematical Sciences \\ University of Bath, Bath \\ BA2 7AY, UK
}

\begin{abstract}
We study radial solutions of the equations of isotropic elasticity in two dimensions (for a disc) and three dimensions (for a sphere). We describe a numerical scheme for computing the critical boundary displacement for cavitation based on the solution of a sequence of initial value problems for punctured domains. We give examples for specific materials and compare our numerical computations with some previous analytical results. A key observation in the formulation of the method is that the strong-ellipticity condition implies that the specification of the normal component of the Cauchy stress on an inner pre-existing but small cavity, leads to a relation for the radial strain as a function of the circumferential strain. To establish the convergence of the numerical scheme we prove a monotonicity property for the inner deformed radius for punctured balls.
\end{abstract}

Key words: nonlinear elasticity, calculus of variations, cavitation, computation.

\section{Introduction}

The phenomenon of void formation in bodies under tension has been observed in laboratory experiments by $[8]$ and others. (See also [7] for a review on cavitation in rubber.) Ball [2] showed, in the context of nonlinear elasticity, that void formation or "cavitation" can decrease the (potential) energy of a body in tension when the tension is sufficiently large.

Consider a ball of nonlinearly elastic material occupying the region $B$ (the unit ball in $\mathbb{R}^{n}, n=2$ or 3 ) in its reference state. Radially symmetric deformations of $B$ are

*pnm@math.uprh.edu

$\dagger$ †j@maths.bath.ac.uk 
deformations $\mathbf{u}: B \rightarrow \mathbb{R}^{n}$ of the form

$$
\mathbf{u}(\mathbf{x})=r(R) \frac{\mathbf{x}}{|\mathbf{x}|}, R=|\mathbf{x}|, r:[0,1] \rightarrow[0, \infty)
$$

satisfying the boundary condition

$$
\mathbf{u}(\mathbf{x})=\lambda \mathbf{x} \text { for } \mathbf{x} \in \partial B
$$

or equivalently,

$$
r(1)=\lambda,
$$

where $\lambda>0$ is given. The energy associated with each deformation is given by

$$
E(\mathbf{u})=\int_{B} W(\nabla \mathbf{u}(\mathbf{x}) d \mathbf{x}
$$

where $W: M_{+}^{n \times n} \rightarrow \mathbb{R}$ is the stored energy function of the material and $M_{+}^{n \times n}$ denotes the set of real $n \times n$ matrices with positive determinant. If $W$ is frame indifferent and isotropic then it is well known that there is a symmetric function $\Phi$ such that

$$
W(\mathbf{F})=\Phi\left(v_{1}, \ldots, v_{n}\right),
$$

where $v_{1}, \ldots, v_{n}$ are the singular values of the matrix $\mathbf{F}$. A simple class of stored energy functions to which the analysis in [2] and the results in this paper can be applied is given by

$$
\Phi\left(v_{1}, \ldots, v_{n}\right)=\kappa \sum_{i=1}^{n} v_{i}^{\alpha}+h\left(v_{1} v_{2} \ldots v_{n}\right) \text { for } \mathbf{F} \in M_{+}^{n \times n},
$$

where $\kappa>0, \alpha \in[1, n)$ and $h:(0, \infty) \rightarrow(0, \infty)$ is a convex compressibility term which satisfies $h(\delta) \rightarrow \infty$ and $\frac{h(\delta)}{\delta} \rightarrow \infty$ as $\delta \rightarrow 0, \infty$ respectively. However, we stress that many of the results and methods of this paper can be applied (or extended) to much more general stored energy functions.

Ball [2] showed that there exists a critical value $\lambda_{\text {crit }}$ such that for $\lambda \leq \lambda_{\text {crit }}$ the radial minimizer is the homogeneous deformation $\mathbf{u} \equiv \lambda \mathbf{x}$, whilst for $\lambda>\lambda_{\text {crit }}$ the radial minimizer is singular. For each $\lambda>\lambda_{\text {crit }}$, this singular minimizer is the unique minimizer in the class of radial deformations and corresponds to a deformation of the form (1) satisfying $r(0)>0$. This deformation therefore produces a spherical hole of radius $c=r(0)$ at the centre of the initially perfect ball (the phenomenon of cavitation). ${ }^{1}$ This symmetric problem has been well studied, there is extensive literature, and we now have

\footnotetext{
${ }^{1}$ The intuitive reason as to why cavitation occurs for such stored energy functions lies in the competition between the two terms that comprise the stored energy function (5) when evaluated on deformations satisfying (2). The first term in the energy $\kappa \sum_{i=1}^{n} v_{i}^{p}$ corresponds to a convex function of $\mathbf{F}=\nabla \mathbf{u}$. Therefore, by Jensen's Inequality, the integral of this term over $B$ would be globally minimized by the homogeneous deformation $\mathbf{u} \equiv \lambda \mathbf{x}$. However, the second (compressibility) term $h\left(v_{1} \ldots v_{n}\right)$ corresponds to the function $h(\operatorname{det} \nabla \mathbf{u})$. The integral over $B$ of this term alone can be minimised by a deformation
} 
a fairly complete understanding of the radial problem (see [13] for a nice survey of results on radial cavitation in nonlinear elasticity). Additional results on radial cavitation can be found, for example, in [1], [35], [25], [26],[15], [6], [12], [14], [27], [17], [29], [24], [21], [34]. General results on the existence and properties of cavitating equilibria in nonlinear elasticity with no assumption of radial symmetry are contained in [16], [30], [31], [32]. (See [33] for an overview of some of these approaches.)

If $c=g(\lambda) \geq 0$ is the radius of the cavity produced by the radial energy minimizer satisfying the boundary condition (3), then a typical bifurcation diagram of cavity radius versus $\lambda$ is shown in figure 1.

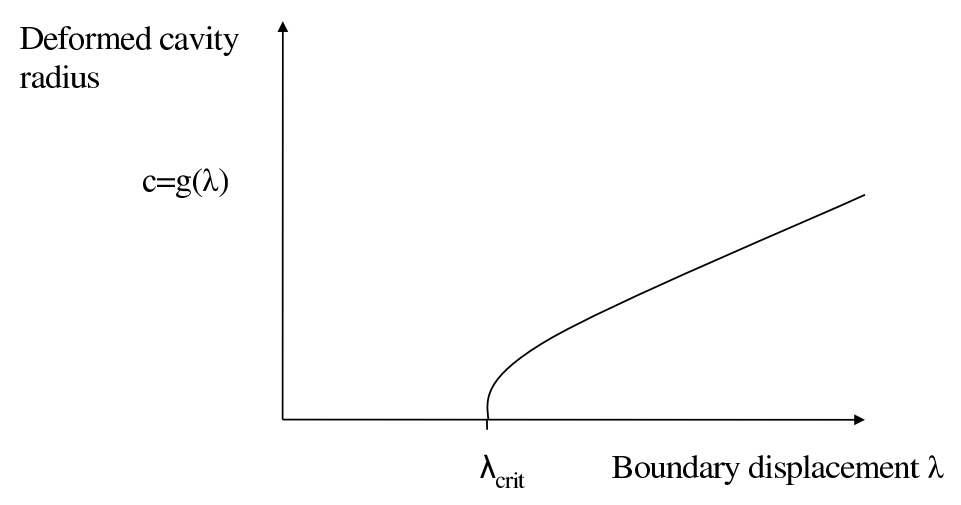

Figure 1: Bifurcation diagram: the graph of $g(\lambda)$.

As cavitation can point to the initiation of fracture or rupture in a body, the computation or characterization of the critical boundary displacement $\lambda_{\text {crit }}$ at which cavitation occurs (together with the corresponding bifurcation diagram) is important from the point of view of design. To underpin the numerical method developed in this paper we first obtain new results on the asymptotic behavior of the function $g(\lambda)$ (these results are used to obtain the convergence rate of the numerical scheme). In particular, we prove

for which $\operatorname{det} \nabla \mathbf{u}$ is close to $d_{0}$ where the pointwise minimum of $h(d)$ is achieved at $d_{0}$. For example

$$
\mathbf{u}(\mathbf{x})=\left(d_{0}|\mathbf{x}|^{n}+\left(\lambda^{n}-1\right)\right)^{\frac{1}{n}} \frac{\mathbf{x}}{|\mathbf{x}|}
$$

satisfies det $\nabla \mathbf{u} \equiv d_{0}$ and produces a hole at the centre of the deformed ball for $\lambda>1$. Now, using the growth assumptions, it follows that for large values of $\lambda$ it is energetically preferable to lower the contribution from the second term by introducing a discontinuity. 
that the function $g$ is monotone increasing, concave and satisfies

$$
\begin{aligned}
g(\lambda) & =\left(\frac{\lambda-\lambda_{\text {crit }}}{A}\right)^{1 / n}+o\left(\left(\lambda-\lambda_{\text {crit }}\right)^{1 / n}\right), \quad \text { as } \lambda \searrow \lambda_{\text {crit }}, \\
g^{\prime}(\lambda) & =\frac{1}{n A}\left(\frac{\lambda-\lambda_{\text {crit }}}{A}\right)^{-(n-1) / n}+o\left(\left(\lambda-\lambda_{\text {crit }}\right)^{-(n-1) / n}\right), \quad \text { as } \lambda \searrow \lambda_{\text {crit }},
\end{aligned}
$$

where $A>0$ is a constant. (Hence, in particular, the curve representing $g(\lambda)$ in figure 1 intersects the $\lambda$ axis with an infinite slope.)

This problem of characterizing $\lambda_{\text {crit }}$ has been studied extensively in the past, see, for example, the works of [10], [5], [36], [11], and [23]. However, the emphasis in most of these papers is in deriving exact, closed form solutions for the cavitation solution for specific materials from which the critical boundary displacement can then be obtained (an exception is the work of [36] which gives interesting bounds on $\lambda_{\text {crit }}$ for stored energy functions with a special structure). In [19] a numerical scheme for the two-dimensional problems is given that uses discrete Fourier transforms in the circumferential direction and finite differences in the radial direction, and that can be used in the radial case to approximate the critical boundary displacement of cavitation. However, due to the sharp boundary layer in the radial strain near the inner cavity, this method requires very refined meshes close to the inner cavity to achieve reasonable precision. ${ }^{2}$ In this paper we describe a numerical scheme for computing the critical boundary displacement and bifurcation diagram for cavitation that applies to a very general class of compressible materials satisfying the strong ellipticity condition. The method is based on the solution of a sequence of initial value problems, thus allowing for the use of commercially available software that can solve these types of problems very efficiently. We give examples for specific materials and compare our numerical computations with some previous analytical results.

The basis of our numerical method to calculate $\lambda_{\text {crit }}$ and the corresponding bifurcation diagram is to approximate by considering radial deformations of punctured balls $B_{\epsilon}=$ $\{\mathbf{x}|\epsilon<| \mathbf{x} \mid<1\}$ of the form

$$
\mathbf{u}(\mathbf{x})=r_{\epsilon}(R) \frac{\mathbf{x}}{|\mathbf{x}|}, R=|\mathbf{x}|, r:[\epsilon, 1] \rightarrow[0, \infty)
$$

satisfying the boundary condition

$$
\mathbf{u}(\mathbf{x})=\lambda \mathbf{x} \text { for } \mathbf{x} \in \partial B \Leftrightarrow r_{\epsilon}(1)=\lambda .
$$

On the inner boundary of $B_{\epsilon}$ we impose the natural boundary condition that the normal stress on this surface is zero. If we plot the deformed radius of the inner cavity of $B_{\epsilon}$, we then obtain the diagram given in figure 2 for small $\epsilon$ (see [12], [25]). Analytic results in [25] show that the curve corresponding to the punctured ball equilibrium solution $r_{\epsilon}$ uniformly converges to that for the solid ball as $\epsilon \rightarrow 0$.

\footnotetext{
${ }^{2}$ See also [3], [4], and [18] for alternative numerical approaches for computing singular minimizers.
} 


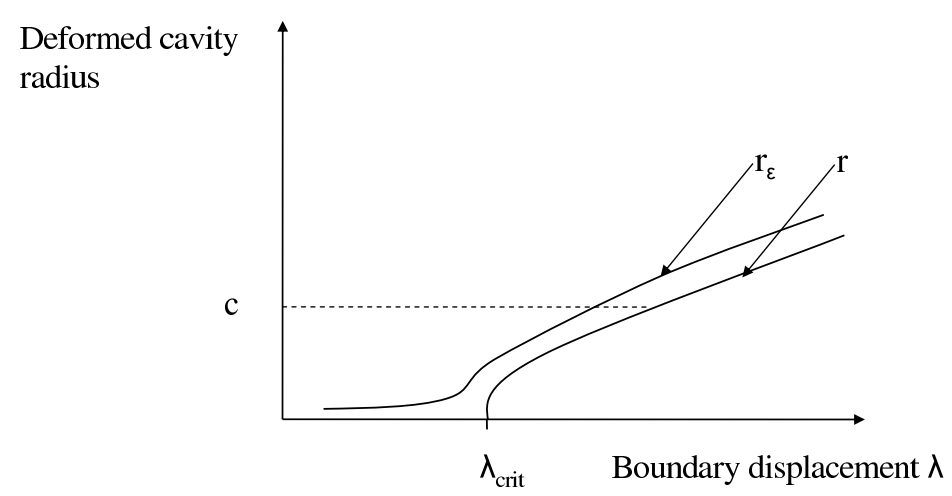

Figure 2: Deformed cavity radius vs boundary displacement for a punctured ball

The idea is to fix the deformed radius $c$ of the inner cavity of $B_{\epsilon}$ which corresponds to fixing the boundary condition $r_{\epsilon}(\epsilon)=c$ (a constant) and then use the zero traction condition to obtain $r_{\epsilon}^{\prime}(\epsilon)$ as a function of $c$ and $\epsilon$. This enables us to determine $r_{\epsilon}(c, R)$ by solving an initial value problem for the radial equilibrium equation (26) and avoids the numerical boundary layer difficulties in trying to solve by shooting from the outer boundary (as in the analytic theory of [35]). Once we obtain $r_{\epsilon}(c, 1)$, we then take the limit as $\epsilon \rightarrow 0$ (see figure 2). Repeating this procedure for a range of values of $c$ yields the bifurcation curve for the solid ball shown in figure 2 . We refer to this method as the inverse method.

After the formulation of the problem and basic constitutive assumptions in Section 2, we briefly review in Section 3 some of the basic results on the existence of radial cavitated solutions. In Proposition 3.2 we obtain new sharp asymptotic estimates on radial cavitated solutions that are required to establish the convergence rate of the proposed numerical scheme, and on the curve of cavity size vs displacement near the critical boundary displacement of cavitation (Proposition 3.3). In Section 4 we consider the mixed displacement-zero traction problem for punctured balls as an approximation of the original problem. After reviewing some basic results for this problem, we extend in Proposition 4.3 a monotonicity property for the inner deformed radius for punctured balls. This result together with Proposition 4.9 forms the basis for the numerical scheme, which we call the inverse method, described in Section 5. A key observation in the formulation of the inverse method is that the specification of the normal component of the Cauchy stress on the inner pre-existing cavity, leads to a relation for the radial strain as 
a function of the circumferential strain on the inner cavity (cf. (63), (66)).

Finally, in Section 6 we give some examples where we apply the inverse method to compute the critical boundary displacement for cavitation. We give examples for Ogden-type materials and compare our numerical computations with previous analytical results, in particular those in [6], [12], [37], [11], and [27]. We present as well some computed figures of the cavity size vs boundary displacement curves, and we illustrate the boundary layer behavior in the derivative of the radial solution and the determinant of the corresponding deformation gradient. A reader interested only on the numerical aspects of the paper may want to concentrate on Section 2 for notation and problem formulation, Theorems 3.1 and 4.1 for the existence results on cavitated solutions and radial equilibria on punctured domains, and Sections 5 and 6 for the numerical method itself and examples.

\section{Formulation of the problem}

We consider a body which in its reference configuration occupies the region

$$
\mathcal{B}=\left\{\mathbf{x} \in \mathbb{R}^{n}:|\mathbf{x}|<1\right\}
$$

where $n=2,3$ and $|\cdot|$ denotes the Euclidean norm. Let $\mathbf{u}: \mathcal{B} \rightarrow \mathbb{R}^{n}$ denote a deformation of the body and let its deformation gradient be

$$
\nabla \mathbf{u}(\mathbf{x})=\frac{\mathrm{d} \mathbf{u}}{\mathrm{d} \mathbf{x}}(\mathbf{x})
$$

For smooth deformations, the requirement that $\mathbf{u}(\mathbf{x})$ is locally invertible and preserves orientation takes the form

$$
\operatorname{det} \nabla \mathbf{u}(\mathbf{x})>0, \quad \mathbf{x} \in \mathcal{B}
$$

Let $W: M_{+}^{n \times n} \rightarrow \mathbb{R}$ be the stored energy function of the material of the body where $M_{+}^{n \times n}=\left\{\mathbf{F} \in M^{n \times n}: \operatorname{det} \mathbf{F}>0\right\}$ and $M^{n \times n}$ denotes the space of real $\mathrm{n}$ by $\mathrm{n}$ matrices. Since we are interested in modelling large deformations, we assume that the stored energy function $W$ satisfies that $W \rightarrow \infty$ as either $\operatorname{det} \mathbf{F} \rightarrow 0^{+}$or $|\mathbf{F}| \rightarrow \infty$. The total energy stored in the body due to the deformation $\mathbf{u}$ is given by

$$
E(\mathbf{u})=\int_{\mathcal{B}} W(\nabla \mathbf{u}(\mathbf{x})) \mathrm{d} \mathbf{x} .
$$

We consider the problem of determining the equilibrium configuration of the body that satisfies (12) and minimizes (13) among all functions belonging to an appropriate Sobolev space and satisfying the boundary condition:

$$
\mathbf{u}(\mathbf{x})=\lambda \mathbf{x}, \quad \mathbf{x} \in \partial \mathcal{B}
$$

where $\lambda>0$ is given. 
If the material is homogeneous and $W$ is isotropic and frame indifferent then it follows that

$$
W(\mathbf{F})=\Phi\left(v_{1}, \ldots, v_{n}\right), \quad \mathbf{F} \in M_{+}^{n \times n},
$$

for some function $\Phi: \mathbb{R}_{+}^{n} \rightarrow \mathbb{R}$ symmetric in its arguments, where $v_{1}, \ldots, v_{n}$ are the eigenvalues of $\left(\mathbf{F}^{t} \mathbf{F}\right)^{1 / 2}$ known as the principal stretches.

For ease of exposition, we consider the special class of Ogden materials ${ }^{3}$ for which:

$$
\Phi\left(v_{1}, \ldots, v_{n}\right)=\kappa \sum_{i=1}^{n} v_{i}^{\alpha}+h\left(v_{1} v_{2} \cdots v_{n}\right),
$$

where $^{4} \kappa>0,1 \leq \alpha<n$ and the function $h$ is convex and satisfies

$$
h(d) \rightarrow \infty, \quad d \rightarrow 0+, \quad \text { or } \quad d \rightarrow \infty
$$

However, we stress that many of the results and methods of this paper can be applied to much more general stored energy functions by combining the analytical results on radial cavitation of [2], [35], [25], [26], [14], [15].

Note that (16) satisfies

$$
\Phi\left(v_{1}, \ldots, v_{n}\right)=\Phi\left(v_{\sigma(1)}, \ldots, v_{\sigma(n)}\right),
$$

where $\sigma$ is any permutation of $\{1, \ldots, n\}$. This condition is equivalent to the material of the body been isotropic. Also, one can easily check that (16) satisfies the inequality:

$$
\frac{\Phi_{, i}\left(v_{1}, \ldots, v_{n}\right)-\Phi_{, j}\left(v_{1}, \ldots, v_{n}\right)}{v_{i}-v_{j}}+\Phi_{, i j}\left(v_{1}, \ldots, v_{n}\right) \geq 0,
$$

for all $i \neq j, v_{i} \neq v_{j}$.

We restrict now to the special case in which the deformation $\mathbf{u}(\cdot)$ is radially symmetric, i.e.,

$$
\mathbf{u}(\mathbf{x})=r(R) \frac{\mathbf{x}}{R}, \quad \mathbf{x} \in \mathcal{B}
$$

for some scalar function $r(\cdot)$, where $R=|\mathbf{x}|$. In this case one can easily check that

$$
v_{1}=r^{\prime}(R) \quad, \quad v_{2}=\cdots=v_{n}=\frac{r(R)}{R}
$$

Thus (13) reduces to

$$
E(\mathbf{u})=\omega_{n} I(r)=\omega_{n} \int_{0}^{1} R^{n-1} \Phi\left(r^{\prime}(R), \frac{r(R)}{R}, \ldots, \frac{r(R)}{R}\right) \mathrm{d} R
$$

\footnotetext{
${ }^{3}$ This stored energy function is a special case of a class proposed by Ogden [20] and is used to model rubber. The Ogden materials include as special cases the Mooney-Rivlin and neo-Hookean materials.

${ }^{4}$ The case $\alpha>n$ is not considered in this paper because any deformation for which the energy (13) is finite would be continuous by the Sobolev Embedding Theorem and thus cavitation is not possible in such a setting. In the case $\alpha=n$, it can also be shown that minimizers with finite energy must be continuous.
} 
where $\omega_{n}=2 \pi$ or $4 \pi$ if $n=2$ or 3 respectively (in general $\omega_{n}$ is area of the unit sphere in $\left.\mathbb{R}^{n}\right)$.

In accord with (12) we have the inequalities

$$
r^{\prime}(R), \frac{r(R)}{R}>0, \quad 0<R<1
$$

and (14) reduces to:

$$
r(1)=\lambda
$$

\section{$3 \quad$ Existence and properties of cavitating solutions}

In this section we review some results concerning the existence and uniqueness of solutions for the problem of minimizing (22) subject to (23) and (24). We also discuss some properties of these equilibrium solutions, in particular those relating to the zero traction boundary condition when a hole opens at the centre of the deformed ball (cf. (29)). We also study the asymptotic behavior of extended cavitating solutions (in Proposition 3.2) and present properties of the bifurcation diagram of cavity size versus displacement (see Proposition 3.3).

Let

$$
\mathcal{A}_{\lambda}=\left\{r \in W^{1,1}(0,1): r(1)=\lambda, r^{\prime}(R)>0 \text { a.e. for } R \in(0,1), r(0) \geq 0\right\} .
$$

The next result is shown in Ball [2] and Sivaloganathan [25] for a more general class of stored energy functions than (16).

Theorem 3.1. The functional (22) attains its infimum over the set $\mathcal{A}_{\lambda}$. If $r$ is a minimizer of $I$ over $\mathcal{A}_{\lambda}$, then it is unique and satisfies:

i) $r^{\prime}(R)>0$ for $0<R \leq 1$,

ii) $r \in C^{2}((0,1])$ and satisfies the radial equilibrium equation:

$$
\frac{\mathrm{d}}{\mathrm{d} R}\left[R^{n-1} \Phi_{, 1}(r(R))\right]=(n-1) R^{n-2} \Phi_{, 2}(r(R)), \quad 0<R<1,
$$

subject to (24) and $r(0) \geq 0$, where:

$$
\Phi_{, i}(r(R))=\Phi_{, i}\left(r^{\prime}(R), \frac{r(R)}{R}, \ldots, \frac{r(R)}{R}\right) \quad i=1, \ldots, n .
$$

Moreover, there exists a $\lambda_{\text {crit }}>1$ such that if $\lambda \leq \lambda_{\text {crit }}$ then

$$
\mathbf{u}_{h}(\mathbf{x})=\lambda \mathbf{x}, \quad \mathbf{x} \in \overline{\mathcal{B}},
$$


is the unique global minimizer of I over $\mathcal{A}_{\lambda}$ and, if $\lambda>\lambda_{\text {crit }}$, then the global minimizer satisfies $r(0)>0$ and the natural boundary condition

$$
\lim _{R \rightarrow 0^{+}} T(r(R))=0
$$

where

$$
T(r(R))=\left(\frac{R}{r(R)}\right)^{n-1} \Phi_{, 1}(r(R)) .
$$

The condition (29) states that if a hole opens at the centre (i.e., if $r(0)>0$ ), then the component of the Cauchy stress normal to the surface of the hole must be zero. This phenomena of void formation is called cavitation and $\lambda_{\text {crit }}$ is called the critical boundary displacement for cavitation.

Using $r \in C^{2}((0,1])$, one can expand (26) to obtain

$$
r^{\prime \prime}(R)=\frac{n-1}{R}\left(\frac{r(R)}{R}-r^{\prime}(R)\right)\left[\frac{\Phi_{, 2}(r(R))-\Phi_{, 1}(r(R))}{r(R) / R-r^{\prime}(R)}+\Phi_{, 12}(r(R))\right] .
$$

From the results in [2], [25] (see, e.g., Proposition 1.6 in [25]) it follows that $r(R) / R-r^{\prime}(R)$ is either constant over $[0,1]$ or never vanishes. Thus, since $r(0)>0$ for a cavitating solution, it follows that this difference is always positive. Hence we can conclude from (31) and the inequality (19) that

$$
r^{\prime \prime}(R) \geq 0, \quad R \in(0,1] .
$$

Thus it follows in particular that $r^{\prime}(R)$ is bounded.

For the stored energy function (16), condition (29) reduces to:

$$
\lim _{R \rightarrow 0^{+}}\left[\alpha\left(r^{\prime}(R)\right)^{\alpha-1}\left(\frac{R}{r(R)}\right)^{n-1}+h^{\prime}\left(r^{\prime}(R)\left(\frac{r(R)}{R}\right)^{n-1}\right)\right]=0 .
$$

Thus, since $r^{\prime}(\cdot)$ is bounded, if $r(0)>0$ we obtain

$$
\lim _{R \rightarrow 0^{+}} r^{\prime}(R)\left(\frac{r(R)}{R}\right)^{n-1}=d_{0}, \quad h^{\prime}\left(d_{0}\right)=0 .
$$

If $r(R)$ is a cavitating solution of $(26),(29)$, then it can be extended to $(0, \infty)$ as a solution. (See e.g., Proposition 2.1, [28].) Moreover, we have the following refinement of Proposition 2.3 in [28]:

Proposition 3.2. Let $r \in C^{2}(0, \infty)$ be a cavitating solution of $(26)$, (29). Then

$$
\begin{aligned}
\frac{r(R)}{R} & =\lambda_{\text {crit }}+\frac{A}{R^{n}}+o\left(R^{-n}\right), \quad \text { as } \quad R \rightarrow \infty, \\
r^{\prime}(R) & =\lambda_{\text {crit }}-(n-1) \frac{A}{R^{n}}+o\left(R^{-n}\right), \quad \text { as } \quad R \rightarrow \infty,
\end{aligned}
$$

where $\lambda_{\text {crit }}$ is as in Theorem 3.1 and $A$ is a positive constant. 
Proof: The proof of this result is similar to that of Proposition 2.3, [28], but keeping an additional term in the required Taylor expansions. The change of variables

$$
\mathrm{e}^{s}=R, \quad v(s)=\frac{r(R)}{R},
$$

transforms equation (26) to the autonomous equation:

$$
\begin{aligned}
\frac{\mathrm{d}}{\mathrm{d} s} \Phi_{, 1}(\dot{v}(s)+v(s), v(s), v(s))= & (n-1)\left(\Phi_{, 2}(\dot{v}(s)+v(s), v(s), v(s))\right. \\
& \left.-\Phi_{, 1}(\dot{v}(s)+v(s), v(s), v(s))\right)
\end{aligned}
$$

where $\dot{v}(s)=\mathrm{d} v(s) / \mathrm{d} s$. For any solution $v(s)$ we have that

$$
\frac{\mathrm{d} \dot{v}}{\mathrm{~d} v}=G(v, \dot{v})
$$

where

$$
\begin{aligned}
G(v, \dot{v})= & (n-1)\left[\Phi_{, 11}(\dot{v}+v, v, \ldots, v)\right]^{-1}\left[\int _ { 0 } ^ { 1 } \left(\Phi_{, 21}(t \dot{v}+v, v, \ldots, v)\right.\right. \\
& \left.\left.-\Phi_{, 11}(t \dot{v}+v, v, \ldots, v)\right) \mathrm{d} t-\Phi_{, 12}(\dot{v}+v, v, \ldots, v)\right]-1
\end{aligned}
$$

The cavitating solution $r(R)$ of (26), (29) is transformed via the change of variables (35) into a solution $\dot{v}=f(v)$ of (37) satisfying $f\left(\lambda_{\text {crit }}\right)=0$. We expand $f$ in a Taylor series about $\lambda_{\text {crit }}$ up to terms of order three:

$$
f(v)=-n\left(v-\lambda_{\text {crit }}\right)+\frac{1}{2} f^{\prime \prime}\left(\lambda_{\text {crit }}\right)\left(v-\lambda_{\text {crit }}\right)^{2}+O\left(\left(v-\lambda_{\text {crit }}\right)^{3}\right),
$$

where upon differentiating $G(v, f(v))$ with respect to $v$ and setting $v=\lambda_{\text {crit }}$, we obtain

$f^{\prime \prime}\left(\lambda_{\text {crit }}\right)=-\frac{n(n-1)}{2 \Phi_{, 11}\left(\lambda_{\text {crit }}, \lambda_{\text {crit }}, \ldots, \lambda_{\text {crit }}\right)}\left[\Phi_{, 111}\left(\lambda_{\text {crit }}, \lambda_{\text {crit }}, \ldots, \lambda_{\text {crit }}\right)-\Phi_{, 112}\left(\lambda_{\text {crit }}, \lambda_{\text {crit }}, \ldots, \lambda_{\text {crit }}\right)\right]$.

It follows now that

$$
\dot{v}(s)=-n\left(v(s)-\lambda_{c r i t}\right)+\frac{1}{2} f^{\prime \prime}\left(\lambda_{c r i t}\right)\left(v(s)-\lambda_{c r i t}\right)^{2}+O\left(\left(v(s)-\lambda_{c r i t}\right)^{3}\right),
$$

or with $u(s)=v(s)-\lambda_{\text {crit }}$, that

$$
\dot{u}(s)=-n u(s)+\frac{1}{2} f^{\prime \prime}\left(\lambda_{c r i t}\right) u^{2}(s)+O\left(u^{3}(s)\right) .
$$

We recall that by Proposition 2.1 in [28], $u(s) \rightarrow 0+$ as $s \rightarrow \infty$. The change of variables $y(s)=1 / u(s)$ transforms the equation above into:

$$
\dot{y}(s)=n y(s)-\frac{1}{2} f^{\prime \prime}\left(\lambda_{c r i t}\right)+E(1 / y(s))
$$


where the error term $E$ satisfies $\frac{E(\delta)}{\delta}$ is uniformly bounded for $\delta \neq 0$ sufficiently small. Integrating the linear part of this equation and putting the resulting expression in terms of $u(s)$, we obtain

$$
\frac{1}{u(s)}=\left[\frac{1}{u\left(s_{0}\right)}-\frac{1}{2 n} f^{\prime \prime}\left(\lambda_{\text {crit }}\right)+\int_{s_{0}}^{s} \mathrm{e}^{-n\left(t-s_{0}\right)} E(u(t)) \mathrm{d} t\right] \mathrm{e}^{n\left(s-s_{0}\right)}+\frac{1}{2 n} f^{\prime \prime}\left(\lambda_{\text {crit }}\right),
$$

for any $s_{0}$. Since $u(s) \rightarrow 0$ as $s \rightarrow \infty$, the integral in this expression is bounded as $s \rightarrow \infty$, and it follows that

$$
\frac{1}{u(s)}=C \mathrm{e}^{n s}+o\left(\mathrm{e}^{n s}\right), \quad s \rightarrow \infty
$$

where

$$
C=\left[\frac{1}{u\left(s_{0}\right)}-\frac{1}{2 n} f^{\prime \prime}\left(\lambda_{c r i t}\right)+\int_{s_{0}}^{\infty} \mathrm{e}^{-n\left(t-s_{0}\right)} E(u(t)) \mathrm{d} t\right] \mathrm{e}^{-n s_{0}} .
$$

Upon differentiating with respect to $s_{0}$, one can easily check that $C$ is independent of $s_{0}$. Moreover, since $u(s) \rightarrow 0+$ as $s \rightarrow \infty$, the bracketed term in the expression for $C$ is positive for $s_{0}$ sufficiently large. Hence by (39)

$$
\frac{1}{\mathrm{e}^{n s} u(s)}=C+o(1), \quad s \rightarrow \infty
$$

and it thus follows that

$$
v(s)-\lambda_{c r i t}=u(s)=A \mathrm{e}^{-n s}+o\left(\mathrm{e}^{-n s}\right), \quad s \rightarrow \infty,
$$

where $A=1 / C$. Using the change of variables (35) we now obtain

$$
\frac{r(R)}{R}=\lambda_{\text {crit }}+\frac{A}{R^{n}}+o\left(R^{-n}\right), \quad R \rightarrow \infty .
$$

Since

$$
r^{\prime}\left(\mathrm{e}^{s}\right)=\dot{v}(s)+v(s)
$$

it follows from (38) that

$$
\begin{aligned}
r^{\prime}\left(\mathrm{e}^{s}\right)-\lambda_{\text {crit }} & =-(n-1) u(s)+\frac{1}{2} f^{\prime \prime}\left(\lambda_{\text {crit }}\right) u^{2}(s)+O\left(u^{3}(s)\right), \\
& =-(n-1) A \mathrm{e}^{-n s}+o\left(\mathrm{e}^{-n s}\right), \quad s \rightarrow \infty
\end{aligned}
$$

i.e.,

$$
r^{\prime}(R)=\lambda_{\text {crit }}-2 \frac{A}{R^{n}}+o\left(R^{-n}\right), \quad R \rightarrow \infty
$$

as required. 
We now explore some consequences of this proposition. The inequality (32) is now valid over $(0, \infty)$. Since for any $\gamma>0$, the function $\gamma r(R / \gamma)$ is also a cavitating solution, we may assume that $r(0)=1$. Thus any other cavitating solution $\hat{r}(R)$ with $\hat{r}(0)=c$, can be written as:

$$
\hat{r}(R)=\operatorname{cr}\left(\frac{R}{c}\right), \quad R \geq 0 .
$$

In particular, setting $R=1$, we obtain

$$
\lambda(c) \equiv \hat{r}(1)=\operatorname{cr}\left(\frac{1}{c}\right), \quad c>0 .
$$

This function gives the boundary condition at $R=1$ as a function of the cavity size. This function is the one that the numerical scheme we describe in Section 5 computes. Note that from the asymptotic expansion (34a) it follows that

$$
\lambda(c)=\lambda_{\text {crit }}+A c^{n}+o\left(c^{n}\right), \quad c \searrow 0,
$$

and thus $\lambda(c)$ is defined and continuous over $[0, \infty)$.

From (40) we obtain

$$
\lambda^{\prime}(c)=r\left(\frac{1}{c}\right)-\frac{1}{c} r^{\prime}\left(\frac{1}{c}\right)=\frac{1}{c}\left[\frac{r\left(\frac{1}{c}\right)}{1 / c}-r^{\prime}\left(\frac{1}{c}\right)\right] .
$$

This expression together with (34b) shows that

$$
\lambda^{\prime}(c)=n A c^{n-1}+o\left(c^{n-1}\right), \quad \text { as } c \searrow 0,
$$

and since $r(R) / R-r^{\prime}(R)>0$ for all $R$, that

$$
\lambda^{\prime}(c)>0, \quad c>0, \quad \lambda^{\prime}(0)=0 .
$$

Moreover

$$
\lambda^{\prime \prime}(c)=\frac{1}{c^{3}} r^{\prime \prime}\left(\frac{1}{c}\right) \geq 0,
$$

by the inequality $(32)$ over $(0, \infty)$, and thus it follows that $\lambda(c)$ is convex.

Since $\lambda^{\prime}(c)>0, c>0$, it follows that $\lambda(c)$ is invertible. If we let $c=g(\lambda), \lambda \geq \lambda_{\text {crit }}$ be its inverse, then by the properties of $\lambda(c)$ stated above, we have the following:

Proposition 3.3. Let $c=g(\lambda), \lambda \geq \lambda_{\text {crit }}$, be the inverse function of $\lambda(c)$. Then $g(\lambda)$ is strictly increasing, concave, and

$$
\begin{aligned}
g(\lambda) & =\left(\frac{\lambda-\lambda_{\text {crit }}}{A}\right)^{1 / n}+o\left(\left(\lambda-\lambda_{\text {crit }}\right)^{1 / n}\right), \quad \text { as } \lambda \searrow \lambda_{\text {crit }}, \\
g^{\prime}(\lambda) & =\frac{1}{n A}\left(\frac{\lambda-\lambda_{\text {crit }}}{A}\right)^{-(n-1) / n}+o\left(\left(\lambda-\lambda_{\text {crit }}\right)^{-(n-1) / n}\right), \quad \text { as } \lambda \searrow \lambda_{\text {crit }} .
\end{aligned}
$$


Proof: That $g$ is strictly increasing and concave follows upon differentiation of the expression $g(\lambda(c))=c$ twice with respect to $c$ and using that $\lambda^{\prime}(c)>0$ and $\lambda^{\prime \prime}(c) \geq 0$ for $c>0$.

From (41) we have that

$$
\frac{\lambda-\lambda_{\text {crit }}}{A}=c^{n}(1+o(1)), \quad c \searrow 0
$$

where $o(1)$ is a quantity that goes to zero as $c \searrow 0$ or equivalently as $\lambda \searrow \lambda_{\text {crit }}$. Equation (43a) follows now from this expression upon setting $c=g(\lambda)$, the identities:

$$
\frac{1}{1+x}=1+O(x), \quad \sqrt[n]{1+x}=1+O(x), \quad x \rightarrow 0
$$

and that $O(o(1))=o(1)$.

Similarly from (42) we obtain:

$$
\frac{1}{g^{\prime}(\lambda(c))}=\lambda^{\prime}(c)=n A c^{n-1}(1+o(1)), \quad c \searrow 0 .
$$

Using the first of the identities (44) and upon setting $c=g(\lambda)$, it follows that

$$
g^{\prime}(\lambda)=\frac{1}{n A g^{n-1}(\lambda)}(1+o(1)), \quad \lambda \searrow \lambda_{\text {crit }}
$$

Equation (43a) can be written as:

$$
g(\lambda)=\left(\frac{\lambda-\lambda_{\text {crit }}}{A}\right)^{1 / n}(1+o(1)), \quad \text { as } \lambda \searrow \lambda_{\text {crit }} .
$$

Equation (43b) follows now from this expression, equation (45), the first of the identities (44), and that $(1+o(1))^{n-1}=1+o(1)$.

Thus the function $g(\lambda)$ intersects the $\lambda$ axis with an infinite slope proportional to $\left(\lambda-\lambda_{\text {crit }}\right)^{-(n-1) / n}$ as $\lambda \searrow \lambda_{\text {crit }}$. (See Figure 1.) For a particular example of the function $g(\lambda)$ satisfying (43) but corresponding to a stored energy similar to (16) with $\alpha=1$, $n=3$, and including a term quadratic in the principal stretches, we refer to [11], equation (29), page 285 .

Example 3.4. Consider the stored energy function studied in [22] (with $\mu$ set equal to 1 for simplicity), i.e.

$$
\Phi\left(v_{1}, v_{2}, v_{3}\right)=\left(v_{1}^{2}+v_{2}^{2}+v_{3}^{2}\right)+h\left(v_{1} v_{2} v_{3}\right)
$$

where

$$
h(d)=\left(a d^{2}-2(a+1) d F+b\right)
$$


for $d \geq 1$ and $a, b \geq 0$ are constants ${ }^{5}$. From expressions (1.7), (1.8) in [22] it follows that the unique radial cavitation solution $r(R)$ with $r(1)=\lambda, r(0)=c$, satisfies

$$
P(\lambda) R^{-3}=\int_{\lambda_{\text {crit }}}^{\frac{r(R)}{R}}\left[1+a \theta^{4}\right]^{\frac{1}{2}} d \theta
$$

where

$$
P(\lambda):=\int_{\lambda_{\text {crit }}}^{\lambda}\left[1+a \theta^{4}\right]^{\frac{1}{2}} d \theta .
$$

Differentiating (47) with respect to $R$, then multiplying by $R^{4}$ and evaluating the resulting expression at $R=0$ yields

$$
P(\lambda)=\frac{1}{3} r(0)^{3} \sqrt{a}=\frac{1}{3} c^{3} \sqrt{a}
$$

Hence by (48)

$$
c=g(\lambda)=\left(\frac{3}{\sqrt{a}} \int_{\lambda_{\text {crit }}}^{\lambda}\left[1+a \theta^{4}\right]^{\frac{1}{2}} d \theta\right)^{\frac{1}{3}} .
$$

It can be verified from (49) and the arguments used in the proof of Proposition 3.3 that (43a) and (43b) hold in this example.

\section{Solutions with a pre--existing hole}

The numerical scheme for computing the critical boundary displacement for cavitation that we describe in Section (5), is based on the solution of a sequence of initial value problems for punctured domains. Thus we consider the situation in which the reference configuration is replaced by the punctured ball:

$$
\mathcal{B}_{\varepsilon}=\left\{\mathbf{x} \in \mathbb{R}^{n}: \varepsilon<|\mathbf{x}|<1\right\},
$$

where $0<\varepsilon<1$. The corresponding radial deformations are given by:

$$
\mathbf{u}_{\varepsilon}(\mathbf{x})=r_{\varepsilon}(R) \frac{\mathbf{x}}{R}, \quad \mathbf{x} \in \mathcal{B}_{\varepsilon} .
$$

The variational problem now is to minimize:

$$
I_{\varepsilon}\left(r_{\varepsilon}\right)=\int_{\varepsilon}^{1} R^{n-1} \Phi\left(r_{\varepsilon}^{\prime}(R), \frac{r_{\varepsilon}(R)}{R}, \ldots, \frac{r_{\varepsilon}(R)}{R}\right) \mathrm{d} R,
$$

subject to:

$$
r_{\varepsilon}^{\prime}(R), \frac{r_{\varepsilon}(R)}{R}>0, \quad \varepsilon<R<1,
$$

\footnotetext{
${ }^{5}$ The definition of $h(d)$ can be extended to $d \in(0,1)$ in any way provided that the resulting $h$ is smooth, convex and satisfies (17).
} 
and the boundary condition:

$$
r_{\varepsilon}(1)=\lambda
$$

Let

$$
\mathcal{A}_{\lambda}^{\varepsilon}=\left\{r \in W^{1,1}(\varepsilon, 1): r(1)=\lambda, r^{\prime}(R)>0 \text { a.e. for } R \in(\varepsilon, 1), r(\varepsilon) \geq 0\right\} .
$$

The following result is proved in [25], again for a more general class of stored energy functions than (16).

Theorem 4.1. The functional $I_{\varepsilon}$ has a unique global minimizer over the set $\mathcal{A}_{\lambda}^{\varepsilon}$. Moreover, there exists a $\delta(\varepsilon)>0$ such that if $r_{\varepsilon}$ is a global minimizer with $\lambda \in(1-\delta(\varepsilon), \infty)$, then $r_{\varepsilon} \in C^{2}([\varepsilon, 1])$ is a solution of $(26)$ over $(\varepsilon, 1)$, and satisfies:

i) $r_{\varepsilon}^{\prime}(R)>0$ for $R \in[\varepsilon, 1]$,

ii) $r_{\varepsilon}(\varepsilon)>0$,

iii) $T\left(r_{\varepsilon}(\varepsilon)\right)=0$.

The idea that the minimizers of $I_{\varepsilon}$ over $\mathcal{A}_{\lambda}^{\varepsilon}$ converge to those of $I$ over $\mathcal{A}_{\lambda}$ (c.f. (22)) was first noticed by Ball [2]. The proof of the following result is given in [25].

Proposition 4.2. Let $r_{\varepsilon}$ be the unique global minimizer of $I_{\varepsilon}$ over $\mathcal{A}_{\lambda}^{\varepsilon}$ and let $\lambda_{\text {crit }}$ be as in Theorem 3.1. Then

i) for $\lambda \leq \lambda_{\text {crit }}$, we have that

$$
\lim _{\varepsilon \rightarrow 0} \sup _{R \in[\varepsilon, 1]}\left|r_{\varepsilon}(R)-\lambda R\right|=0,
$$

ii) if $\lambda>\lambda_{\text {crit }}$, then we have that

$$
\lim _{\varepsilon \rightarrow 0} \sup _{R \in[\varepsilon, 1]}\left|r_{\varepsilon}(R)-r_{c}(R)\right|=0,
$$

where $r_{c}$ is the cavitating minimizer of I over $A_{\lambda}$.

For the remainder of this paper we will use the following notation for minimizers.

\section{Notation.}

We denote by $r_{\varepsilon}(R, \lambda)$ the global minimizer predicted by Theorem 4.1 and by $r(R, \lambda)$ the corresponding minimizer in Theorem 3.1.

The next proposition will be very important for the numerical scheme that we describe in Section 5. It pertains to certain monotonicity properties for the inner deformed radius for punctured balls. The first part of the proposition is the content of Proposition 4.10 in [25], but part (ii) is a new result. 
Proposition 4.3. The function $r_{\varepsilon}(R, \lambda)$ has the following monotonicity properties:

i) $r_{\varepsilon}\left(\varepsilon, \lambda_{1}\right) \leq r_{\varepsilon}\left(\varepsilon, \lambda_{2}\right)$ for $\lambda_{1} \leq \lambda_{2}$,

ii) $r_{\varepsilon_{1}}\left(\varepsilon_{1}, \lambda\right)<r_{\varepsilon_{2}}\left(\varepsilon_{2}, \lambda\right)$ for $\varepsilon_{1}<\varepsilon_{2}$.

Before we give a proof of this result we need to discuss a transformation of our problem into an autonomous equation thus allowing us to use phase plane techniques to study the functions $r_{\varepsilon}$. Let us recall that the change of variables (35) transforms (26) into the equivalent autonomous equation (36) where $\dot{v}(s)=\mathrm{d} v(s) / \mathrm{d} s$. Since

$$
\Phi_{, 1}(v, v, \ldots, v)=\Phi_{, 2}(v, v, \ldots, v), \quad v>0,
$$

it follows that $v=$ constant is always a solution of (36). Thus the $v$-axis represents a line of critical points of $(36)$ in the phase plane $(v, \dot{v})$ and these are the only critical points. Thus non-constant solutions must satisfy:

i) $\dot{v}(s)>0$ for all $s$ in the domain of existence or

ii) $\dot{v}(s)<0$ for all $s$ in the domain of existence.

Since $r^{\prime}(R)=\dot{v}(s)+v(s)$, it follows from Proposition 1.1 and Corollary 1.2 in [25] that we need to consider only the cases $\dot{v}(s) \leq 0$.

Proof:[ of Proposition 4.3] The first part of the proposition is the content of Proposition 4.10 in [25]. For part (ii), first note that by uniqueness of solutions of the initial value problem for 26 , if $r_{\varepsilon_{1}}^{\prime}(1, \lambda)=r_{\varepsilon_{2}}^{\prime}(1, \lambda)$, then $r_{\varepsilon_{1}}(R, \lambda)=r_{\varepsilon_{2}}(R, \lambda)$ for all $R \in\left[\varepsilon_{2}, 1\right]$. The result would follow now from Property (i) in Theorem (4.1). Assume now that $r_{\varepsilon_{1}}^{\prime}(1, \lambda) \neq r_{\varepsilon_{2}}^{\prime}(1, \lambda)$. Using formula $(2.33)$ in $[25]$ and phase plane considerations, we have that for $\varepsilon_{1}<\varepsilon_{2}$ we must have $r_{\varepsilon_{1}}^{\prime}(1, \lambda)>r_{\varepsilon_{2}}^{\prime}(1, \lambda)$. Hence

$$
r_{\varepsilon_{1}}(R, \lambda)<r_{\varepsilon_{2}}(R, \lambda), \quad \gamma<R<1,
$$

for some $\gamma \in\left[\varepsilon_{2}, 1\right)$. If $r_{\varepsilon_{1}}(\bar{R}, \lambda)=r_{\varepsilon_{2}}(\bar{R}, \lambda)$ for some $\bar{R} \in[\gamma, 1)$, then again formula (2.33) in [25] and phase plane considerations lead us to conclude that $r_{\varepsilon_{1}}^{\prime}(\bar{R}, \lambda)>r_{\varepsilon_{2}}^{\prime}(\bar{R}, \lambda)$. But this would contradict (56). Hence $\gamma=\varepsilon_{2}$ in (56) which implies in particular that

$$
r_{\varepsilon_{1}}\left(\varepsilon_{2}, \lambda\right)<r_{\varepsilon_{2}}\left(\varepsilon_{2}, \lambda\right) .
$$

Since $r_{\varepsilon_{1}}(R, \lambda)$ is increasing over $\left[\varepsilon_{1}, 1\right]$, we have that

$$
r_{\varepsilon_{1}}\left(\varepsilon_{1}, \lambda\right)<r_{\varepsilon_{1}}\left(\varepsilon_{2}, \lambda\right)<r_{\varepsilon_{2}}\left(\varepsilon_{2}, \lambda\right) .
$$

We now study some consequences of these monotonicity properties. In particular the next two lemmas establish the existence of punctured and cavitated solutions with a prescribed cavity size. In Proposition 4.6 we show that the punctured solution is always smaller than the cavitated solution for the same inner cavity size on the interval $[\varepsilon, 1]$. 
Lemma 4.4. For any $c \geq \varepsilon>0$, there exists a unique $\lambda$ such that $r_{\varepsilon}(\varepsilon, \lambda)=c$.

Proof: Using the Implicit Function Theorem one can show that for the stored energy function (16), the equation $\Phi_{, 1}(r, v, \ldots, v)=0$ has a unique solution $r=\phi(v)$, for a smooth $\phi:(0, \infty) \rightarrow(0, \infty)$. By phase plane considerations for $(36)$, it follows that there exists a unique curve $(v(s), \dot{v}(s))$ in the phase plane, passing through $(c / \varepsilon, \phi(c / \varepsilon)-c / \varepsilon)$ and converging to the point $\left(\lambda^{*}, 0\right)$ as $s \rightarrow \infty$. The time map function to the left of equation (2.33) in [25] is a monotone decreasing function of $\lambda \in\left(\lambda^{*}, c / \varepsilon\right]$ from $+\infty$ down to zero. Thus equation (2.33) in [25] has a unique solution $\lambda$ that yields a total time of $\log (1 / \varepsilon)$. For this $\lambda$ by construction, $r_{\varepsilon}(\varepsilon, \lambda)=c$.

Lemma 4.5. For any $c>0$ there exists a unique $\lambda>\lambda_{\text {crit }}$ such that $r(0, \lambda)=c$.

Proof: This follows from Theorem 1.11, part (iv), in [25].

Proposition 4.6. For any $c \geq \varepsilon>0$,

$$
r_{\varepsilon}(R, \lambda) \leq r(R, \bar{\lambda}), \quad R \in[\varepsilon, 1]
$$

where $r_{\varepsilon}(\varepsilon, \lambda)=c$ and $r(0, \bar{\lambda})=c$.

Proof: That $r(R, \bar{\lambda})$ and $r_{\varepsilon}(R, \lambda)$ exist follows from Lemmas 4.4 and 4.5. Moreover, since both $r(R, \bar{\lambda})$ and $r_{\varepsilon}(R, \lambda)$ are equal to $c$ at $R=0$ and $R=\varepsilon$ respectively, and both are strictly increasing, it follows that

$$
r(\varepsilon, \bar{\lambda})>r_{\varepsilon}(\varepsilon, \lambda)
$$

Suppose for a contradiction that the graphs of $r(R, \bar{\lambda})$ and $r_{\varepsilon}(R, \lambda)$ intersect at $R_{0} \in(\varepsilon, 1]$. Let

$$
v_{2} \equiv \frac{r\left(R_{0}, \bar{\lambda}\right)}{R_{0}}=\frac{r_{\varepsilon}\left(R_{0}, \lambda\right)}{R_{0}}
$$

Consider now the graphs of $r(R, \bar{\lambda})$ and $r_{\varepsilon}(R, \lambda)$ against $R$. Define $v_{1}=c / \varepsilon$ and consider the line through the origin of slope $v_{1}$. This line intersects $r(R, \bar{\lambda})$ at $R_{1} \in(\varepsilon, 1]$ and $r_{\varepsilon}(R, \lambda)$ at $R=\varepsilon$. Hence

$$
\frac{r\left(R_{1}, \bar{\lambda}\right)}{R_{1}}=\frac{r_{\varepsilon}(\varepsilon, \lambda)}{\varepsilon}=v_{1} .
$$

Then looking at the time map in the phase plane for each of the corresponding solution curves we obtain a contradiction, since the cavitating solution curve should pass from $v=v_{1}$ to $v=v_{2}$ in a longer time than that for the $r_{\varepsilon}$ curve, but the interval $\left[\varepsilon, R_{0}\right]$ is longer than the interval $\left[R_{1}, R_{0}\right]$.

The next two results are concerned with limiting processes. In particular, in Lemma 4.7 we establish the convergence of the inner cavity radius for punctured solutions to 
the corresponding inner cavity radius for the problem in the full domain, with $\lambda$ fixed and the inner hole $\varepsilon \rightarrow 0$. Then in Proposition 4.8 we show that for a fixed cavity size $c>0$ but with the inner hole $\varepsilon \rightarrow 0$, the corresponding boundary displacements for the punctured solutions converge to the boundary displacement for the problem in the full domain.

Lemma 4.7. Let $\left(\varepsilon_{k}\right)$ be a monotone decreasing sequence converging to zero. Then for any $\lambda>0$, the sequence $\left(r_{\varepsilon_{k}}\left(\varepsilon_{k}, \lambda\right)\right)$ is a monotone decreasing sequence with

$$
\lim _{k \rightarrow \infty} r_{\varepsilon_{k}}\left(\varepsilon_{k}, \lambda\right)=\left\{\begin{array}{cc}
r(0, \lambda) & , \lambda>\lambda_{\text {crit }}, \\
0, & \lambda \leq \lambda_{\text {crit }} .
\end{array}\right.
$$

Proof: This follows from Propositions 4.2 and 4.3, and the continuity of $r(\cdot, \lambda)$.

Proposition 4.8. Let $\left(\varepsilon_{k}\right)$ be a monotone decreasing sequence converging to zero and let $c>0$. Let $r_{\varepsilon_{k}}\left(R, \lambda_{k}\right)$ be such that $r_{\varepsilon_{k}}\left(\varepsilon_{k}, \lambda_{k}\right)=c$ for all $k$. Then the sequence $\left(\lambda_{k}\right)$ is monotone increasing with $\lambda_{k} \rightarrow \bar{\lambda}$, where $r(R, \bar{\lambda})$ is such that $r(0, \bar{\lambda})=c$.

Proof: The existence of $r_{\varepsilon_{k}}\left(R, \lambda_{k}\right)$ for $\varepsilon_{k}$ sufficiently small follows from Lemma 4.4 and that of $r(R, \bar{\lambda})$ follows from Lemma 4.5. To argue by contradiction, suppose that for some $k$,

$$
\lambda_{k+1} \leq \lambda_{k}, \quad \varepsilon_{k+1}<\varepsilon_{k} .
$$

Then we have that

$$
\begin{aligned}
c=r_{\varepsilon_{k+1}}\left(\varepsilon_{k+1}, \lambda_{k+1}\right) & \leq r_{\varepsilon_{k+1}}\left(\varepsilon_{k+1}, \lambda_{k}\right), \quad \text { (Proposition 4.3, part (i)) } \\
& <r_{\varepsilon_{k}}\left(\varepsilon_{k}, \lambda_{k}\right), \quad \text { (Proposition 4.3, part (ii)) } \\
& =c,
\end{aligned}
$$

which is a contradiction. Hence $\lambda_{k}<\lambda_{k+1}$ for all $k$.

To prove the convergence of $\left(\lambda_{k}\right)$, first note that

$$
r_{\varepsilon_{k}}\left(\varepsilon_{k}, \lambda_{k}\right)=c=r(0, \bar{\lambda})<r_{\varepsilon_{k}}\left(\varepsilon_{k}, \bar{\lambda}\right),
$$

where for the last inequality we used Lemma 4.7. Since $r_{\varepsilon_{k}}\left(\varepsilon_{k}, \cdot\right)$ is increasing by Proposition 4.3, part (i), it must follow that $\lambda_{k} \leq \bar{\lambda}$. Hence $\lambda_{k} \nearrow \lambda^{*} \leq \bar{\lambda}$. If $\lambda^{*}<\bar{\lambda}$, then

$$
r\left(0, \lambda^{*}\right)<r(0, \bar{\lambda})=c .
$$

(We used here that as a consequence of Corollary 1.2 and Theorem 1.11 in [25], the function $r(0, \cdot)$ is a continuous monotone function of $\lambda$ that is strictly increasing for $\lambda>\lambda_{\text {crit. }}$.) However, since $\lambda_{k}<\lambda^{*}$,

$$
c=r_{\varepsilon_{k}}\left(\varepsilon_{k}, \lambda_{k}\right)<r_{\varepsilon_{k}}\left(\varepsilon_{k}, \lambda^{*}\right) \rightarrow r\left(0, \lambda^{*}\right)<c,
$$


which is a contradiction. Hence $\lambda^{*}=\bar{\lambda}$.

The next result which is the main one of this section, establishes the convergence (and its rate) of the boundary displacements for punctured solutions to the critical boundary displacement for cavitation of the problem in the full domain, for any given sequence of inner cavity sizes approaching zero.

Proposition 4.9. Let $\left(c_{m}\right)$ be a monotone decreasing sequence converging to zero. Then there exists a monotone sequence $\left(\varepsilon_{m}\right)$ converging to zero, and a sequence $\left(\lambda_{m}\right)$ such that

$$
\lim _{m \rightarrow \infty} \lambda_{m}=\lambda_{\text {crit }}
$$

where $r_{\varepsilon_{m}}\left(\varepsilon_{m}, \lambda_{m}\right)=c_{m}$. Moreover for some constants $K, M>0$,

$$
\left|\lambda_{m}-\lambda_{\text {crit }}\right| \leq K c_{m}^{n}, \quad m>M .
$$

Proof: Since $r(0, \cdot)$ is continuous and $r\left(0, \lambda_{\text {crit }}\right)=0$, for each $\eta>0$ there exists a $\delta>0$ with $\delta<\eta$ such that

$$
r(0, \lambda)<\eta \quad \lambda_{\text {crit }} \leq \lambda<\lambda_{\text {crit }}+\delta .
$$

Let $M>0$ be such that $c_{m}<\eta$ for all $m>M$. Applying Proposition 4.8 to the sequence $(1 / k)$ and $c_{m}$, we get that for each $m$ there exists a monotone increasing sequence $\left(\lambda_{k}^{(m)}\right)$ such that

$$
\lim _{k \rightarrow \infty} \lambda_{k}^{(m)}=\bar{\lambda}_{m}
$$

where $r_{1 / k}\left(1 / k, \lambda_{k}^{(m)}\right)=c_{m}$ and $r\left(0, \bar{\lambda}_{m}\right)=c_{m}$. By the monotonicity of $r(0, \cdot)$ and using (58), since $c_{m}<\eta$ for $m>M$, it follows that

$$
\lambda_{\text {crit }}<\bar{\lambda}_{m}<\lambda_{\text {crit }}+\delta<\lambda_{\text {crit }}+\eta, \quad m>M
$$

Moreover, by the convergence in (59) and the above inequality, it follows that there exists $k(m)$ such that

$$
\lambda_{\text {crit }}<\lambda_{k(m)}^{(m)} \leq \bar{\lambda}_{m}<\lambda_{\text {crit }}+\eta, \quad r_{1 / k(m)}\left(1 / k(m), \lambda_{k(m)}^{(m)}\right)=c_{m}, \quad m>M .
$$

By the monotonicity of $\left(\lambda_{k}^{(m)}\right)$ in $(59)$, we can always choose $k(m)$ such that $k(m)>$ $k(m-1)$. With

$$
\lambda_{m}=\lambda_{k(m)}^{(m)}, \quad \varepsilon_{m}=\frac{1}{k(m)},
$$

we have that $\left(\varepsilon_{m}\right)$ is monotone and converging to zero, and that

$$
\lambda_{\text {crit }}<\lambda_{m} \leq \bar{\lambda}_{m}<\lambda_{\text {crit }}+\eta, \quad r_{\varepsilon_{m}}\left(\varepsilon_{m}, \lambda_{m}\right)=c_{m}, \quad m>M,
$$

from which it follows in particular that $\lambda_{m} \rightarrow \lambda_{\text {crit }}$. Moreover since

$$
r\left(R, \bar{\lambda}_{m}\right)=c_{m} r_{*}\left(\frac{R}{c_{m}}\right),
$$


where $r_{*}(R)$ is the cavitating solution with $r_{*}(0)=1$, it follows that

$$
\bar{\lambda}_{m}=c_{m} r_{*}\left(\frac{1}{c_{m}}\right) .
$$

Thus by the inequality in (60) we get that

$$
\lambda_{\text {crit }}<\lambda_{m} \leq c_{m} r_{*}\left(\frac{1}{c_{m}}\right), \quad m>M
$$

By the asymptotic expression (34a) in Proposition 3.2, we have

$$
c_{m} r_{*}\left(\frac{1}{c_{m}}\right)=\lambda_{c r i t}+A c_{m}^{n}+o\left(c_{m}^{n}\right), \quad m \rightarrow \infty .
$$

This together with the inequality above shows that $\left|\lambda_{m}-\lambda_{\text {crit }}\right|=O\left(c_{m}^{n}\right)$ from which (57) follows.

\section{The numerical scheme}

In this section we describe a very simple numerical scheme for approximating the critical boundary displacement for cavitation $\lambda_{\text {crit }}$. The fundamental idea leading or motivating the construction of the scheme is a consequence of the following observation. Using condition (ii) in Theorem 4.1 and definition (30), it follows that (iii) of Theorem 4.1 is equivalent to:

$$
\Phi_{, 1}\left(r_{\varepsilon}(\varepsilon)\right)=0
$$

For the stored energy function (16), assume that in addition to (17) the function $h(\cdot)$ satisfies:

$$
h^{\prime \prime}(d)>0, \quad h^{\prime}(d) \rightarrow\left\{\begin{aligned}
\infty & , d \rightarrow \infty \\
-\infty & , \quad d \rightarrow 0^{+}
\end{aligned}\right.
$$

The function (16) now satisfies:

$$
\begin{gathered}
\Phi_{, 1}\left(v_{1}, \ldots, v_{n}\right) \rightarrow\left\{\begin{array}{rl}
-\infty, & v_{1} \rightarrow 0^{+}, \\
\infty \quad, & v_{1} \rightarrow \infty
\end{array} \quad v_{2}, \ldots, v_{n}, \quad \text { fixed },\right. \\
\Phi_{, 11}\left(v_{1}, \ldots, v_{n}\right)>0 .
\end{gathered}
$$

It follows upon recalling the notation (27), properties (65), and the Implicit Function Theorem that there exists a smooth function $\phi:(0, \infty) \rightarrow(0, \infty)$ such that $(63)$ is equivalent to

$$
r_{\varepsilon}^{\prime}(\varepsilon)=\phi\left(\frac{r_{\varepsilon}(\varepsilon)}{\varepsilon}\right)
$$


Thus instead of prescribing $\lambda$, we could prescribe the inner cavity $r_{\varepsilon}(\varepsilon)=c$. Using equation (66), we solve now the initial value problem:

$$
\begin{aligned}
& \frac{\mathrm{d}}{\mathrm{d} R}\left[R^{n-1} \Phi_{, 1}\left(r_{\varepsilon}(R)\right)\right]=(n-1) R^{n-2} \Phi_{, 2}\left(r_{\varepsilon}(R)\right), \quad \varepsilon<R<1, \\
& r_{\varepsilon}(\varepsilon)=c \quad, \quad r_{\varepsilon}^{\prime}(\varepsilon)=\phi\left(\frac{c}{\varepsilon}\right) .
\end{aligned}
$$

It follows from Lemma 4.4 that for some $\lambda(c)$ this problem has a unique solution $r_{\varepsilon}(R, \lambda(c))$ defined over the whole of the interval $[\varepsilon, 1]$ and with $r_{\varepsilon}(1, \lambda(c))=\lambda(c)$.

We define the sets:

$$
\begin{aligned}
& \Omega_{\varepsilon}=\left\{\left(\lambda, r_{\varepsilon}(\varepsilon, \lambda)\right): \lambda \geq 1\right\} \\
& \Omega_{0}=\{(\lambda, r(0, \lambda)): \lambda \geq 1\}
\end{aligned}
$$

Note that these definitions can be written equivalently as:

$$
\begin{aligned}
& \Omega_{\varepsilon}=\left\{(\lambda(c), c): c \geq \varepsilon, \quad r_{\varepsilon}(\varepsilon, \lambda(c))=c\right\} \\
& \Omega_{0}=\{(\lambda(c), c): c>0, \quad r(0, \lambda(c))=c\} .
\end{aligned}
$$

The set $\Omega_{\varepsilon}$ can now be approximated by solving the IVP (67) for a given set of values of $c$. This procedure works very efficiently and fast as compared to solving the nonlinear boundary value problem (26), (i)-(iii) of Theorem (4.1) over $(\varepsilon, 1)$. We should point out that for $\varepsilon$ small, the numerical solution of the IVP (67) is not an easy task because of the singular behavior of the circumferential strain $r_{\varepsilon}(R) / R$. In addition the determinant function:

$$
d_{\varepsilon}(R)=\left(\frac{r_{\varepsilon}(R)}{R}\right)^{n-1} r_{\varepsilon}^{\prime}(R),
$$

as well as the derivative $r_{\varepsilon}^{\prime}(R)$, both have very sharp boundary layers close to $R=\varepsilon$ as well. In our numerical experiments we used the MATLAB platform which includes very accurate IVP solvers that can effectively handle these sharp boundary layers, thus allowing us to get very accurate sketches of the sets $\Omega_{\varepsilon}$.

Using Propositions 4.8 and 4.9 it follows that for a set of values of $c$ and $\varepsilon$ progressively becoming very small, we can approximate as well the set $\Omega_{0}$ and the critical boundary displacement $\lambda_{\text {crit }}$. We call this procedure to compute $\Omega_{\varepsilon}, \Omega_{0}$, and $\lambda_{\text {crit }}$ the inverse method. We can describe the general procedure for computing $\lambda_{\text {crit }}$ as follows:

Procedure 5.1. Let $\left(\varepsilon_{k}, c_{k}\right)$ be a sequence converging to $(0,0)$.

1) For $k=0,1,2, \ldots$,

a) Compute an approximate solution $\nu_{k}$ of the equation:

$$
\Phi_{, 1}\left(\nu_{k}, c_{k} / \varepsilon_{k}, \ldots, c_{k} / \varepsilon_{k}\right)=0
$$


b) Compute an approximate solution $r_{k}(R)$ of the initial value problem:

$$
\begin{aligned}
\frac{\mathrm{d}}{\mathrm{d} R}\left[R^{n-1} \Phi_{, 1}\left(r_{k}(R)\right)\right]= & (n-1) R^{n-2} \Phi_{, 2}\left(r_{k}(R)\right), \quad \varepsilon_{k}<R<1, \\
r_{k}\left(\varepsilon_{k}\right)=c_{k} & , \quad r_{k}^{\prime}\left(\varepsilon_{k}\right)=\nu_{k} .
\end{aligned}
$$

c) Set $\lambda_{k}=r_{k}(1)$.

2) Repeat steps (a)-(c), until the sequence $\left\{\lambda_{k}\right\}$ satisfies a certain stopping criteria.

A variation of this procedure in which the value of $c$ is kept fixed while the epsilon progressively becomes smaller, is used to generate points arbitrarily close to the set $\Omega_{0}$, or with $\varepsilon$ fixed and the $c$ becoming progressively smaller, we can approximate $\Omega_{\varepsilon}$.

The convergence result in Proposition 4.9 is for an arbitrary monotone decreasing sequence $\left(c_{k}\right)$, but yields the existence of a corresponding monotone sequence $\left(\varepsilon_{k}\right)$. The rate of convergence of the $\left(\lambda_{k}\right)$ is $O\left(c_{k}^{n}\right)$ where $n$ is the dimension (Prop. (4.9), inequality (57)). This is independent of the sequence $\left(c_{k}\right)$. If the $\left(c_{k}\right)$ converges at a certain specific rate to zero, this rate will be reflected in the convergence of the $\left(\lambda_{k}\right)$ but to the $n$-th power. In practice, the way the $\left(\varepsilon_{k}\right)$ are selected will affect this rate of convergence. To see this, let $\eta_{k}=\lambda_{k}-\bar{\lambda}_{k}$, where $r\left(0, \bar{\lambda}_{k}\right)=c_{k}$. It follows now from equations (61) and (62) in the proof of Proposition 4.9, that

$$
\lambda_{k}-\lambda_{\text {crit }}=\eta_{k}+A c_{k}^{n}+o\left(c_{k}^{n}\right), \quad m \rightarrow \infty .
$$

Thus the actual rate of convergence will depend on how well $\bar{\lambda}_{k}$ is approximated which in turn depends both on $\varepsilon_{k}$ and the accuracy of the IVP solver. In the examples in Section (6), the sequence $\left(c_{k}\right)$ is taken as $c_{k}=10^{-k}$, with $\varepsilon_{k}$ fixed at $10^{-k-4}$ or $10^{-2 k}$.

\section{$6 \quad$ Numerical examples}

In this section we give four examples to which we apply the method described in Section 5 for computing $\lambda_{\text {crit }}$. Two of the examples correspond to stored energy functions that generalise the stored energy function (16) and we also include one which does not satisfy the standard existence theory for radial minimisers. The effectiveness of the proposed method in these examples illustrates its robustness as a numerical scheme.

We implemented ${ }^{6}$ the Procedure 5.1 using MATLAB: for the root finding step we used a linear fractional method (see e.g. [9]) and the ode15s routine for the solution of the IVP's. In Example (6.1) we show plots of the computed $\Omega_{\varepsilon}$ sets, with the corresponding $\Omega_{0}$ set, and of the previously mentioned boundary layers in the determinant (68) and $r_{\varepsilon}^{\prime}(R)$.

\footnotetext{
${ }^{6}$ Some of the MATLAB codes used for the simulations are available at http://math.uprh.edu/ urmaa/invmet.
} 
Example 6.1. In this example we consider the case of (16) in which

$$
h(d)=C d^{\gamma}+D d^{-\delta},
$$

where $C, D \geq 0, \delta>0$, and $\gamma>1$. The function (16) with the function $h(\cdot)$ above, satisfies both (17) and (65). We choose $D$ as

$$
D=\frac{\alpha+\gamma C}{\delta},
$$

which guarantees that the reference configuration is stress-free.

For the case $C=1, \alpha=\gamma=\delta=1.5, \kappa=1$ and $n=3$, we show in Table 1 a sequence of values of $\left(\lambda_{k}\right)$ where $r_{\varepsilon_{k}}\left(\varepsilon_{k}, \lambda_{k}\right)=c_{k}$ as computed by the method described in the previous section. The computed value of the critical boundary displacement is $\lambda_{\text {crit }}=1.148169909$. In Figure 3 we show several of the computed $\Omega_{\varepsilon}$ sets, with the corresponding $\Omega_{0}$ set.

\begin{tabular}{|c|c|c|}
\hline$c_{k}$ & $\varepsilon_{k}$ & $\lambda_{k}$ \\
\hline \hline 0.02 & $2 \mathrm{e}-006$ & 1.148171813 \\
0.01 & $1 \mathrm{e}-006$ & 1.148170147 \\
0.001 & $1 \mathrm{e}-007$ & 1.148169909 \\
0.0001 & $1 \mathrm{e}-008$ & 1.148169909 \\
$1 \mathrm{e}-005$ & $1 \mathrm{e}-009$ & 1.148169909 \\
\hline
\end{tabular}

Table 1: Iterations $\left(\lambda_{k}\right)$ generated by the inverse method and approximating $\lambda_{\text {crit }}$ for example (16), (69) with $C=1, \alpha=\gamma=\delta=1.5$ and $n=3$.

In addition, we show in Figures (4), (5), and (6) plots for the functions $d_{\varepsilon}(R)$ (cf. (68)), $r_{\varepsilon}^{\prime}(R)$, and $r_{\varepsilon}(R)$ respectively for the values of $c=0.5,0.2,0.1,0.01$. The corresponding values of $\varepsilon$ are taken to be $c \times 10^{-4}$. We can appreciate in these pictures the sharp boundary layers close to $R=\varepsilon$ in the functions $d_{\varepsilon}(R)$ and $r_{\varepsilon}^{\prime}(R)$ for $\varepsilon$ small.

Example 6.2. We consider the special case of (70) and (71) in which $\alpha=\beta=1$ and $c_{3}=0$. We write in this case $\Phi$ instead of $\Phi^{k}$ in $(70)$, and $h(d)$ instead of $h(k, d)$ in (71b). This stored energy function was considered in [11] where an exact solution of the boundary value problem (26), (29) with $r(0)>0$ is obtained. In particular it is shown that

$$
\lambda_{\text {crit }}=k_{1}^{1 / 3}, \quad h^{\prime}\left(k_{1}\right)=0 .
$$

For $(71 b)$ (with $c_{3}=0$ ) we have that

$$
k_{1}=\left(\frac{\eta c_{5}}{\delta c_{4}}\right)^{\frac{1}{\delta+\eta}} .
$$


Furthermore, if $\delta=\eta+2$, then the equation $\Phi_{1}(v, \tau, \tau)=0$ can be solved for $v$ in closed form.

For the special case $\delta=3, \eta=1, c_{1}=1.0, c_{2}=1.0, c_{4}=1.0$, and $c_{5}=6$ (chosen so that the undeformed configuration is a natural state) we have that $\lambda_{\text {crit }}=1.059463094$. We show in Table 2 the results of the inverse method showing convergence to the exact critical boundary displacement in this case as well.

\begin{tabular}{|c|c|c|}
\hline$c_{k}$ & $\varepsilon_{k}$ & $\lambda_{k}$ \\
\hline \hline 0.1 & 0.01 & 1.055404103 \\
0.01 & 0.0001 & 1.05904303 \\
0.001 & $1 \mathrm{e}-006$ & 1.059421316 \\
0.0001 & $1 \mathrm{e}-008$ & 1.059459165 \\
$1 \mathrm{e}-005$ & $1 \mathrm{e}-010$ & 1.05946255 \\
\hline
\end{tabular}

Table 2: Iterations $\left(\lambda_{k}\right)$ generated by the inverse method and approximating $\lambda_{\text {crit }}$ for the example in [11].

Example 6.3. In this example we study the behavior of the proposed numerical scheme in a sequence of compressible problems approaching the incompressible limit. In particular we used the stored energy function in [27] given by:

$$
\Phi^{k}\left(v_{1}, v_{2}, v_{3}\right)=\Phi^{\mathrm{inc}}\left(v_{1}, v_{2}, v_{3}\right)+h\left(k, v_{1} v_{2} v_{3}\right)
$$

where

$$
\begin{aligned}
\Phi^{\mathrm{inc}}\left(v_{1}, v_{2}, v_{3}\right) & =c_{1}\left(\sum_{i=1}^{3} v_{i}^{\alpha}-3\right)+c_{2}\left(\sum_{i<j}\left(v_{i} v_{j}\right)^{\beta}-3\right), \\
h(k, d) & =\frac{c_{3}}{k}|d-1|^{\gamma}+c_{4} d^{\delta}+c_{5} d^{-\eta}
\end{aligned}
$$

where $1<\alpha<3,1 \leq \beta<3 / 2, \gamma>1, \delta \geq 1, \eta>0, c_{1}, c_{3}, c_{5}>0$, and $c_{2}, c_{4} \geq 0$ are constants. The condition $\alpha c_{1}+2 \beta c_{2}+\delta c_{4}-\eta c_{5}=0$ guarantees that the undeformed configuration is a natural state for all $k$.

If $\lambda_{c r i t}^{k}$ denotes the critical boundary displacement corresponding to the stored energy function $\Phi^{k}$, then in [27] it is shown that $\left\{\lambda_{\text {crit }}^{k}\right\}$ converges as $k \rightarrow 0$ to the critical boundary displacement for the incompressible case, i.e., $\lambda_{c r i t}^{k} \rightarrow 1$. In the particular example that follows we show that the inverse method captures as well this incompressible limit. In particular for the case:

$$
\begin{array}{ccc}
\alpha=1.5, \quad \beta=1.0, \quad \gamma=2.0, & \delta=1.5, & \eta=1.5, \\
c_{1}=1.0, \quad c_{2}=1.0, \quad c_{3}=1.0, \quad c_{4}=1.0, & c_{5}=10 / 3,
\end{array}
$$

we show in Table 3 the sequence of critical boundary displacements for the compressible problems approaching the incompressible limit of one. 


\begin{tabular}{|c|c||c|c|}
\hline$k$ & $\lambda_{\text {crit }}^{k}$ & $k$ & $\lambda_{\text {crit }}^{k}$ \\
\hline \hline 0.1 & 1.044740301 & $1 \mathrm{e}-005$ & 1.000007904 \\
0.01 & 1.006776275 & $1 \mathrm{e}-006$ & 1.000001358 \\
0.001 & 1.000722242 & $1 \mathrm{e}-007$ & 1.000000703 \\
0.0001 & 1.000073314 & $1 \mathrm{e}-008$ & 1.000000638 \\
\hline
\end{tabular}

Table 3: Iterations $\left(\lambda_{c r i t}^{k}\right)$ generated by the inverse method for the stored energy function (70) in the incompressible limit.

Example 6.4. The following stored energy function is studied in [6], [12], and [37]:

$$
\Phi\left(v_{1}, v_{2}, v_{3}\right)=v_{1}^{-2}+v_{2}^{-2}+v_{3}^{-2}+2 v_{1} v_{2} v_{3} .
$$

Note that this function does not satisfy condition (17) and has $\alpha=-2$. We show in Table 4 a sequence of values of $\left(\lambda_{k}\right)$ where $r_{\varepsilon_{k}}\left(\varepsilon_{k}, \lambda_{k}\right)=c_{k}$ as computed by the Procedure 5.1 described in the previous section. The computed value of the critical boundary displacement is $\lambda_{\text {crit }}=1.30874$, which agrees with the value reported in [12] and [37] to the number of digits shown.

\begin{tabular}{|c|c|c|}
\hline$c_{k}$ & $\varepsilon_{k}$ & $\lambda_{k}$ \\
\hline \hline 0.01 & $1 \mathrm{e}-006$ & 1.308741033 \\
0.001 & $1 \mathrm{e}-007$ & 1.308740619 \\
0.0001 & $1 \mathrm{e}-008$ & 1.308740618 \\
$1 \mathrm{e}-005$ & $1 \mathrm{e}-009$ & 1.308740618 \\
\hline
\end{tabular}

Table 4: Iterations $\left(\lambda_{k}\right)$ generated by the inverse method and approximating $\lambda_{\text {crit }}$ for example (72).

\section{Concluding Remarks}

The inverse method leads nicely to a very efficient method for computing the critical boundary displacement for cavitation for planar and spherical bodies among radial deformations. The basic idea behind this method is that one can solve the equation (63) for the radial strain in terms of the circumferential one (cf. (66)). This idea carries over to the non-radial case provided the stored energy function $W$ is strongly elliptic, i.e. that

$$
\mathbf{a} \otimes \mathbf{b}: \frac{\mathrm{d}^{2} W(\mathbf{F})}{\mathrm{d} \mathbf{F}^{2}}[\mathbf{a} \otimes \mathbf{b}]>0,
$$

for all $\mathbf{a}, \mathbf{b} \in \mathbb{R}^{3} \backslash\{\mathbf{0}\}$ and all $\mathbf{F}$ with $\operatorname{det} \mathbf{F}>0$. 
Our original interest when looking at the problem of computing the critical boundary displacement for cavitation was actually in the computation of the boundary of the quasiconvexity region for non-symmetric $2 \mathrm{~d}$ and $3 \mathrm{~d}$ cavitation. By the above observation, in principle the inverse method could be applied in this scenario as well. That is:

- Using strong ellipticity, one can get all the information of the deformation gradient along the normal direction from that along tangential directions and boundary data on any given surface (the pre-existing hole).

- One can then setup a Cauchy problem to be solved, mimicking in this way the inverse method.

- A general three-dimensional convergence result for nonsymmetric situations, in the spirit of Proposition 4.2, is available (see [34]).

There are two major obstacles to this line of reasoning:

- As opposed to the radial case in which one knows before hand that the deformed surface is spherical and thus can be characterized by a scalar (the radius), in the non-symmetric problem the deformed cavity shape is an arbitrary surface.

- Cauchy problems for elliptic partial differential equations are in general ill-posed.

At present it is unclear as to how one might overcome these issues but this is the subject of ongoing study.

Acknowledgement: The research of Sivaloganathan was sponsored in part by a grant from the Royal Society of London. That of Negrón-Marrero was sponsored in part by the National Security Agency (NSA) under grant number H98230-04-C-0486. 


\section{References}

[1] Antman, S.S. and Negrón-Marrero, P.V., The Remarkable Nature of Radially Symmetric Equilibrium States of Aelotropic Nonlinearly Elastic Bodies, J. Elasticiy, 18, 131-164, 1987.

[2] Ball, J. M., Discontinuous Equilibrium Solutions and Cavitation in Nonlinear Elasticity, Phil. Trans. Royal Soc. London A 306, 557-611, 1982.

[3] Ball, J. M. and Knowles, G., A numerical method for detecting singular minimizers, Numerische Math., 92, 193-204, 1986.

[4] Ball, J.M., Singularities and computation of minimizers for variational problems. In: Foundations of Computational Mathematics, R. DeVore , A. Iserles and E. Suli Eds., London Mathematical Society Lecture Note Series, Vol. 284, pp 1-20. Cambridge University Press, 2001.

[5] Beatty, M.F., A Theory of Elastic Stability for Incompressible Hyperelastic Bodies, Int. J. Solids Struct. 3, 23-37, 1967.

[6] Chung, D-T and Horgan, C.O. and Abeyaratne, R., The finite deformation of internally pressurized hollow cylinders and spheres for a class of compressible elastic materials, Int. J. Solids and Structures, 22, 1557-1570, 1986.

[7] Gent, A.N., Cavitation in Rubber: A Cautionary Tale, Rubber Chemistry and Technology, G49-G53, 1990.

[8] Gent, A. N. and Lindley, P. B., Internal Rupture of Bonded Rubber Cylinders in Tension, Proc. R. Soc. Lond. A 249, 195-205, 1958.

[9] Heath, M. T., Scientific Computing: An Introductory Survey, 2nd Edition, McGrawHill, New York, 2002.

[10] Hill, R., On Uniqueness and Stability in the Theory of Finite Elastic Strain, J. Mech. Phys. Solids 5, 229-241, 1957.

[11] Horgan, C.O., Void nucleation and growth for compressible non-linearly elastic materials: an example, Int. J. Solids Structures, Vol. 29, No. 3, 279-291, 1992.

[12] Horgan, C.O., and Abeyaratne, R., A bifurcation problem for a compressible nonlinearly elastic medium growth of a micro-void, J. Elasticity, 16, 189-200, 1986.

[13] Horgan, C.O. and Polignone, D.A., Cavitation in Nonlinearly Elastic Solids: A Review, Appl. Mech. Rev., Vol. 48, No. 8, 1995.

[14] James, R.D. and Spector, S.J., The Formation of Filamentary Voids in Solids, J. Mech. Phys. Solids, 39, 783-814, 1991. 
[15] F. Meynard, Existence and nonexistence results on the radially symmetric cavitation problem, Quarterly of Applied Mathematics, Vol 50, no. 2, 201-226, 1992.

[16] S. Müller and S. J. Spector, An existence theory for nonlinear elasticity that allows for cavitation, Arch. Rational Mech. Anal., 131, 1-66, 1995.

[17] J.G. Murphy and S. Biwa, Nonmonotonic cavity growth in finite compressible elasticity, Int. J. Solids Structures, 34, 3859-3872,1997.

[18] Negrón-Marrero, P. V., A Numerical Method for Detecting Singular Minimizers of Multidimensional Problems in Nonlinear Elasticity, Numerische Mathematik, 58, 135-144, 1990.

[19] Negrón-Marrero, P. V. and Betancourt O., The Numerical Computation of Singular Minimizers in Two Dimensional Elasticity, Journal of Computational Physics, Vol. 113, No. 2, pp. 291-303, 1994.

[20] Ogden, R. W., Large deformation isotropic elasticity, Proc. Roy. Soc. London, A326:565-584 and A328:567-583, 1972.

[21] Thomas J. Pence and Hungyu Tsai, Swelling-Induced Cavitation of Elastic Spheres, Mathematics and Mechanics of Solids, 11, 527-551, 2006.

[22] Pericak-Spector, K.A. and Sivaloganathan, J. and Spector, S.J., An explicit radial cavitation solution in nonlinear elasticity, Math. and Mech. of Solids, 7: 87-93, 2002.

[23] Polignone, D.A. and Horgan, C.O., Cavitation for Incompressible Anisotropic Nonlinearly Elastic Spheres, Journal of Elasticity, 33, 27-65, 1993.

[24] X. Shang and C. Cheng, Exact solution for cavitated bifurcation for compressible hyperelastic materials, Int. J. of Eng. Sci., 39, 1101-1117, 2001.

[25] Sivaloganathan, J., Uniqueness of regular and singular equilibria for spherically symmetric problems of nonlinear elasticity, Arch. Rat. Mech. Anal., 96, 97-136, 1986.

[26] Sivaloganathan, J., A field theory approach to stability of radial equilibria in nonlinear elasticity, Math. Proc. Camb. Phil. Soc., 99, 586-604, 1986.

[27] Sivaloganathan, J., Cavitation, the incompressible limit, and material inhomogeneity, Quarterly of Applied Mathematics, Vol. XLIX, No. 3, 521-541, 1991.

[28] Sivaloganathan, J., On the stability of cavitating equilibria, Quarterly of Applied Mathematics, Vol. LIII, No. 2, 301-313, 1995.

[29] Sivaloganathan, J., On cavitation and degenerate cavitation under internal hydrostatic pressure, Proc. R. Soc. London A, 455, 3645-3664, 1999. 
[30] Sivaloganathan, J. and Spector, S. J., On the existence of minimizers with prescribed singular points in nonlinear elasticity, J. Elasticity, 59, 83-113, 2000.

[31] Sivaloganathan, J. and Spector, S. J., On the optimal location of singularities arising in variational problems in nonlinear elasticity, Journal of Elasticity, 58: 191-224, 2000 .

[32] Sivaloganathan, J. and Spector, S. J., On cavitation, configurational forces and implications for fracture in a nonlinearly elastic matrial, Journal of Elasticity, 67: 25-49, 2002.

[33] Sivaloganathan, J. and Spector, S. J., A variational approach to fracture of materials, IUTAM Symposium on Asymptotics, Singularities and Homogenisation in Problems of Mechanics (A.B.Movchan ed), Springer, 2004, 295-306.

[34] Sivaloganathan, J., Spector, S.J. and Tilakraj, V., The convergence of regularized minimizers for cavitation problems in nonlinear elasticity, SIAM J. Appl. Math, 66, 736-757, 2006.

[35] Stuart, C.A., Radially symmetric cavitation for hyperelastic materials, Analyse non lineaire, 2, 33-66, 1985.

[36] Stuart, C.A., Estimating the Critical Radius for Radially Symmetric Cavitation, Quarterly of Applied Mathematics, Vol. 13, No. 2, 251-263, 1993.

[37] Tian-hu, H., A theory of the appearance and growth of the micro-spherical void, Int. J. of Fracture, 43, R51-R55, 1990. 


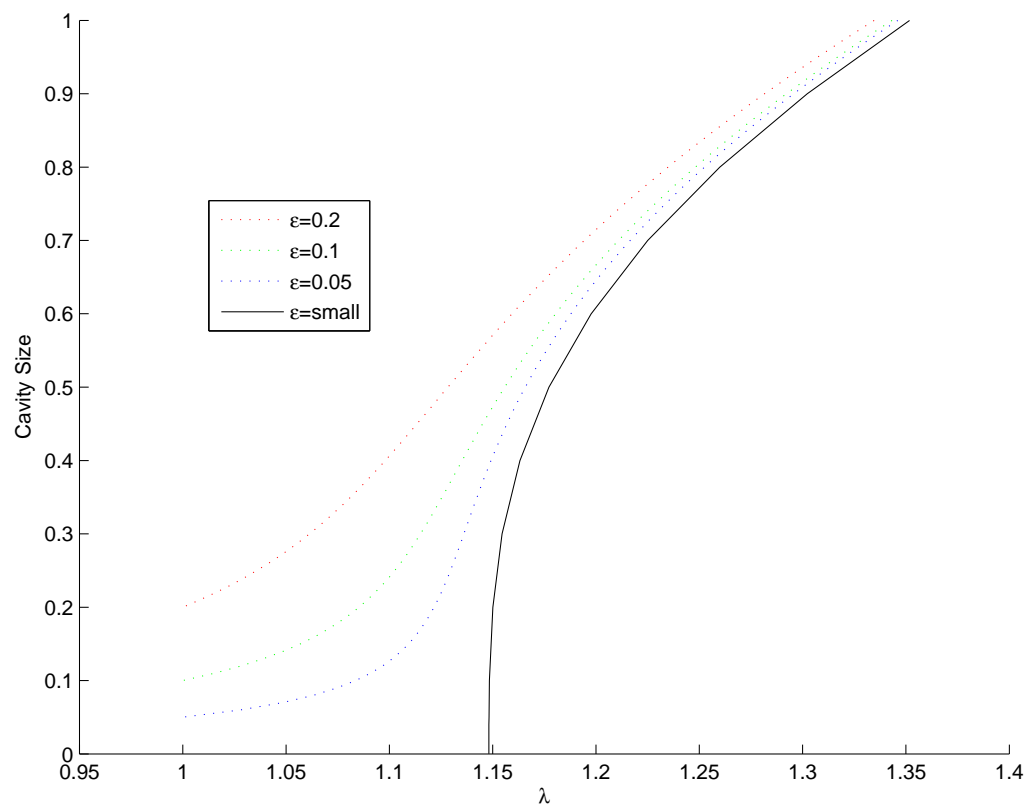

Figure 3: The sets $\Omega_{\varepsilon}$ with $\varepsilon=0.2,0.1,0.05$ and $\Omega_{0}$ for the stored energy function (16), (69) with $C=1, \alpha=\gamma=\delta=1.5$ and $n=3$.

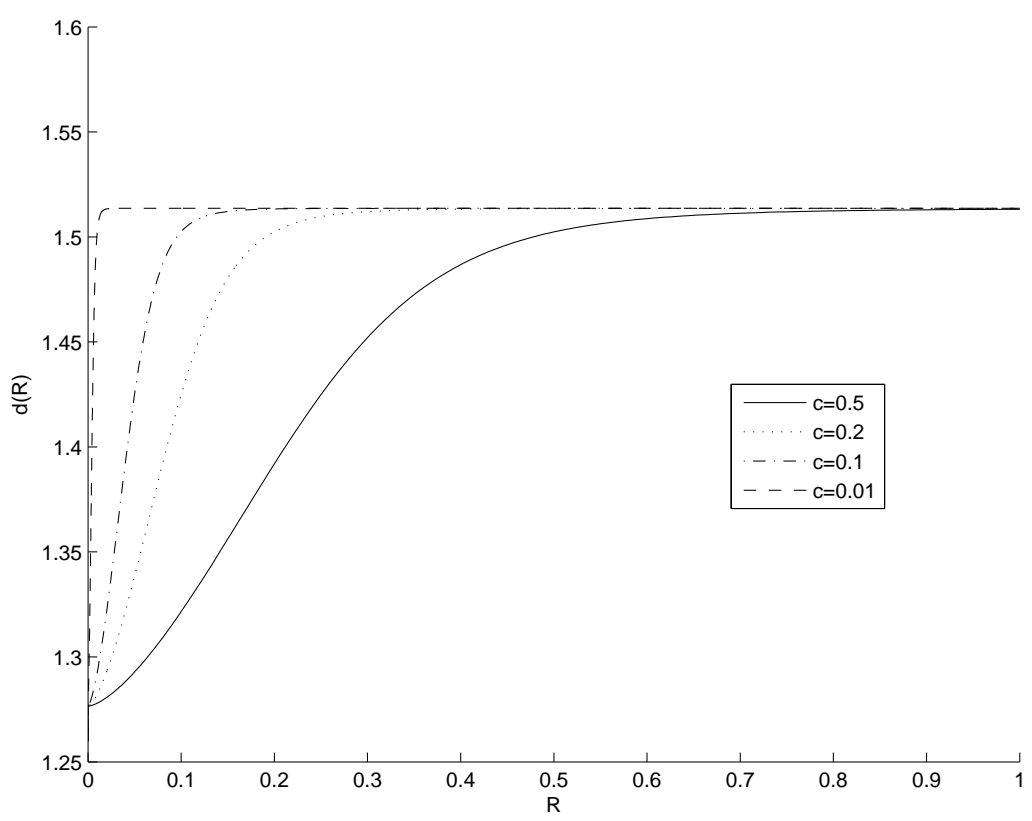

Figure 4: Plots for the function $d_{\varepsilon}(R)$, for the values of $c=0.5,0.2,0.1,0.01$ with corresponding values of $\varepsilon=c \times 10^{-4}$. 


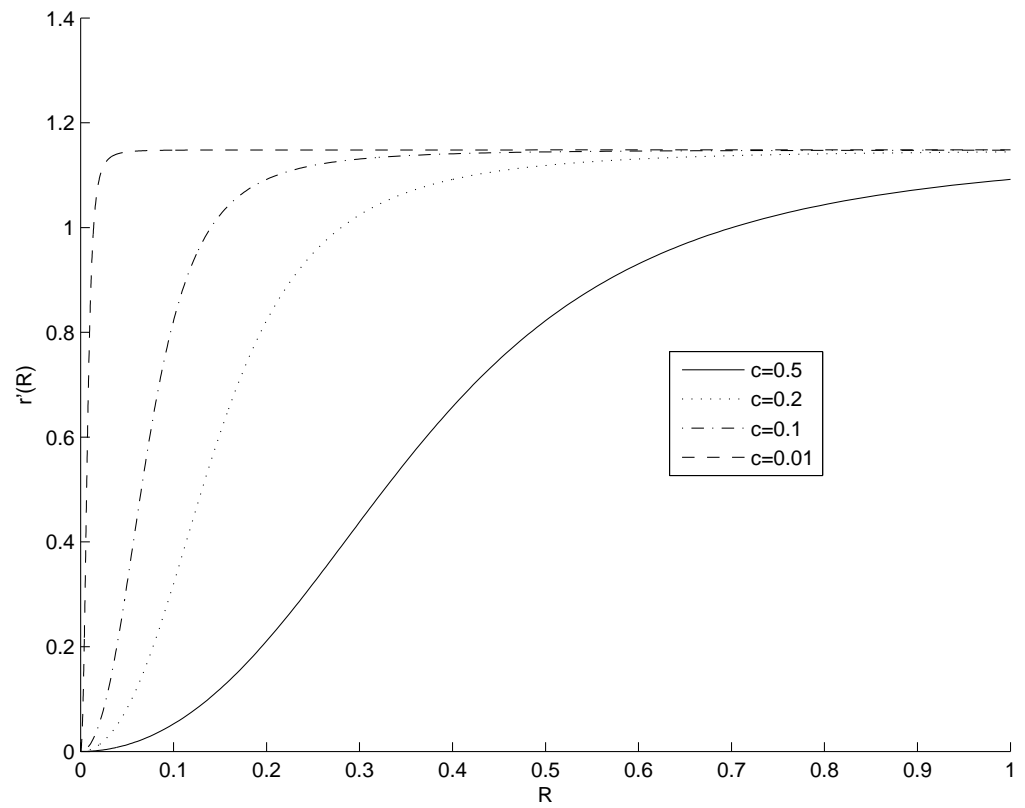

Figure 5: Plots for the function $r_{\varepsilon}^{\prime}(R)$, for the values of $c=0.5,0.2,0.1,0.01$ with corresponding values of $\varepsilon=c \times 10^{-4}$.

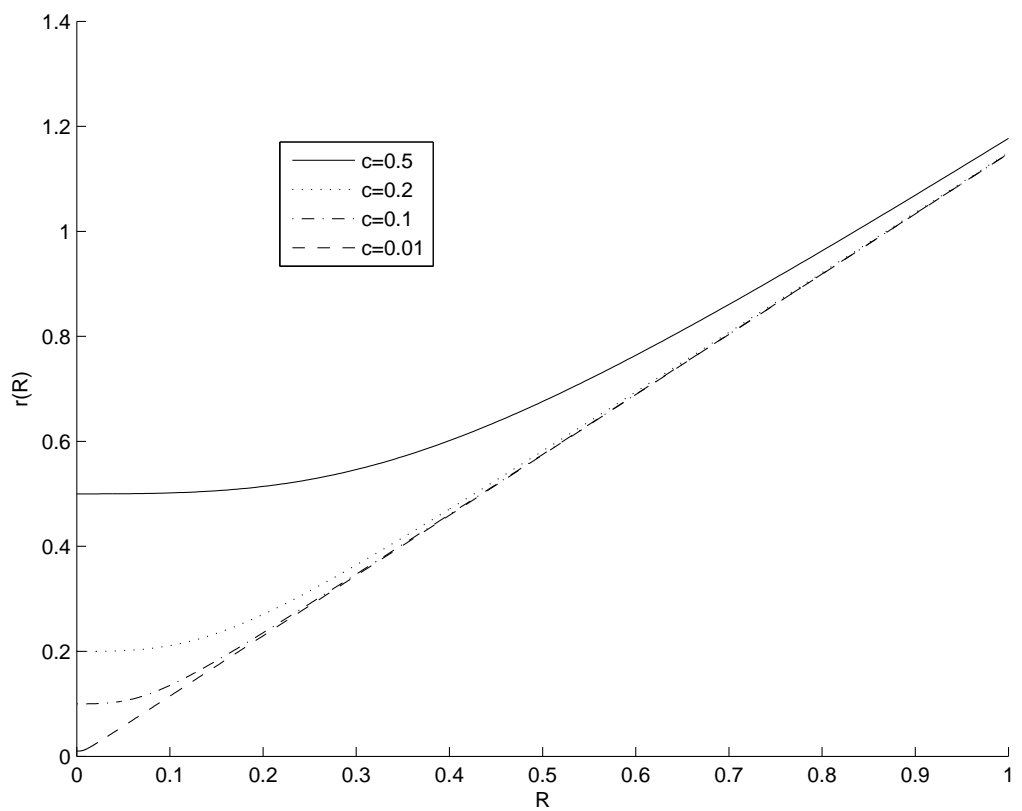

Figure 6: Plots for the function $r_{\varepsilon}(R)$, for the values of $c=0.5,0.2,0.1,0.01$ with corresponding values of $\varepsilon=c \times 10^{-4}$. 